\title{
Miniaturiser les antennes pour la lumière visible
}

Sébastien Bidault ${ }^{(1)}$ (sebastien.bidault@espci.fr) et Nicolas Bonod ${ }^{(2)}$

(1) Institut Langevin, ESPCI Paris, PSL Research University/CNRS, 1 rue Jussieu, 75238 Paris Cedex 05

(2) Institut Fresnel, Aix-Marseille Université/CNRS/Centrale Marseille, 13397 Marseille Cedex 20

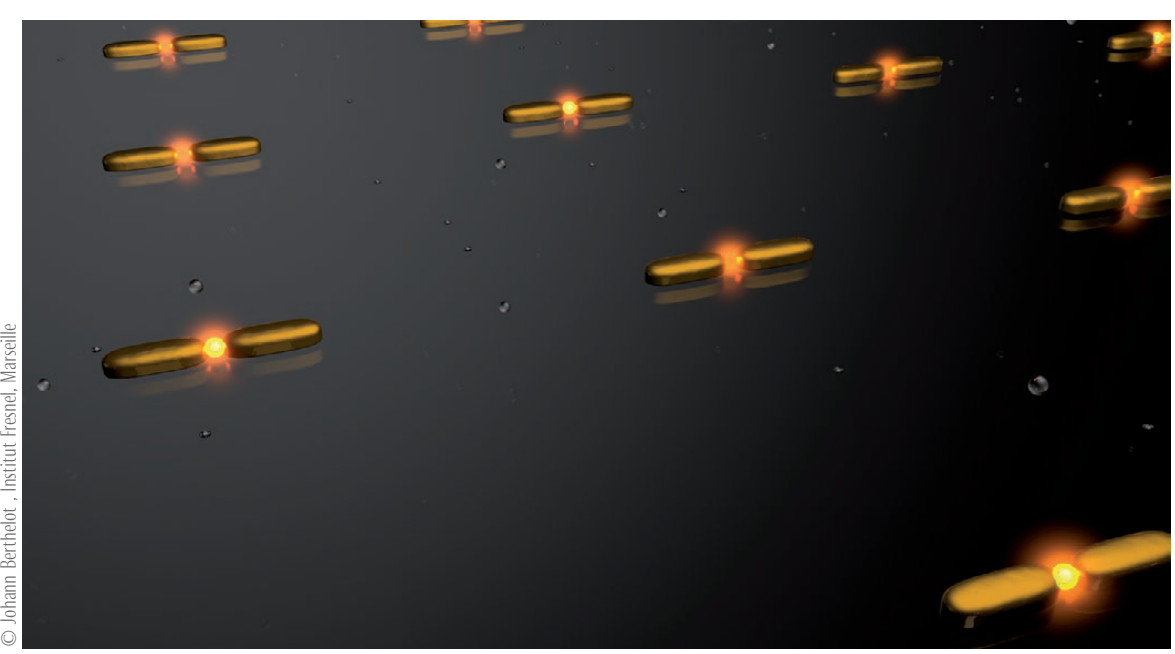

Aux fréquences hertziennes, les antennes permettent d'amplifier le couplage entre un émetteur/récepteur d'ondes électromagnétiques et le champ lointain.

En les miniaturisant pour les adapter à des fréquences optiques un million de fois plus élevées, il est possible de concevoir des antennes pour la lumière capables d'exalter l'absorption ou l'émission de photons par la matière condensée, à des échelles nanométriques et à température ambiante.

Les améliorations constantes des méthodes de nanofabrication, par lithographie ou par auto-assemblage, ont permis d'exploiter ces nano-antennes dans l'optimisation de sources de lumière, mais aussi dans la conception de capteurs et dans l'étude de biomolécules.

Vue d'artiste d'une série d'antennes optiques nanométriques, formées de deux particules d'or de 10 à 100 nanomètres de taille caractéristique, et fabriquées sur un substrat transparent. Ces antennes permettent d’amplifier le champ électromagnétique de plusieurs ordres de grandeur dans l'interstice qui sépare les deux particules, afin d'exalter l'émission de photons de sources de lumière, à l'état solide et à température ambiante, comme des molécules fluorescentes ou des éléments nanométriques de semi-conducteur.

La capacité de la matière à absorber ou émettre des photons est essentiellement dominée par le couplage entre le champ électrique de la lumière et les électrons polarisables du matériau. Dans le cas élémentaire où un photon interagit avec un atome isolé en phase gazeuse, le couplage présente une résonance dont la largeur ne dépasse pas quelques mégahertz. À l'inverse, en matière condensée, le déplacement des électrons est sensible aux vibrations subies par les noyaux atomiques (phonons), entraînant un élargissement spectral des couplages lumière-matière à température ambiante. Ces phénomènes d'élargissement homogène et de décohérence à l'état solide réduisent de plusieurs ordres de grandeur les efficacités d'absorption et d'émission de lumière, et permettent de considérer des émetteurs individuels - tels que des molécules fluorescentes, des phosphores ou des éléments nanométriques de semiconducteur - comme de simples dipôles électriques rayonnant sur une large gamme spectrale et incohérents les uns par rapport aux autres.
C'est alors que l'adaptation du concept d'antenne des micro-ondes vers les longueurs d'onde optiques devient pertinente : en radiofréquence, pour améliorer l'émission ou la réception d'ondes électromagnétiques par une source dipolaire à large bande, on l'associe à des éléments métalliques fortement polarisables, de taille inférieure à la longueur d'onde, qui amplifient son couplage avec le champ lointain [1-3]. Les progrès récents des nanosciences pour structurer les matériaux inorganiques à des échelles bien inférieures aux longueurs d'onde optiques a permis la mise au point de nanoantennes pour la lumière à partir de métaux nobles et, plus récemment, de semi-conducteurs comme le silicium. Ces objets, fortement polarisables, permettent d'amplifier l'interaction entre la lumière et la matière et ainsi d'optimiser des sources de lumière, des photodétecteurs ou des cellules photovoltaïques. De plus, comme la fréquence de résonance de ces antennes est sensible à leur géométrie à des échelles inférieures à la longueur d'onde, elles permettent 


\section{\>}

de mesurer simplement des déformations nanométriques avec des applications, plus inattendues, dans la détection ou l'étude de biomolécules telles que des brins d'ADN ou des protéines.

\section{Modifier les propriétés d'émission d'une source de lumière}

Dans le domaine des fréquences optiques et en matière condensée, un émetteur peut être généralement décrit par trois niveaux d'énergie électroniques quantifiés : un niveau fondamental ; un niveau excité métastable de très courte durée de vie, atteint après absorption d'un photon; et un niveau luminescent de longue durée de vie, atteint après couplage avec les vibrations des noyaux atomiques et pouvant émettre un photon de plus faible énergie en se désexcitant (fig. 1). Jusqu'aux travaux de Purcell en 1946, il était admis que le temps de vie du niveau excité (inverse du taux d'émission $\Gamma$ ) était une constante mais, compte tenu de la règle d'or de Fermi, ce temps dépend directement du couplage entre l'émetteur et son environnement. Le développement de l'électrodynamique quantique en cavité a montré que $\Gamma$ peut être fortement modulé en couplant l'émetteur à une cavité optique dont la fréquence de résonance est proche de la fréquence des photons émis. Dans le cas d'un couplage faible, cette modification peut être prédite aussi bien par des calculs d'électrodynamique quantique que par une approche classique modélisant l'émission d'un dipôle harmonique. Ainsi, le rapport $\Gamma / \Gamma_{\mathrm{o}}$ du taux d'émission $\Gamma$ d'un émetteur quantique dans un environnement complexe par rapport au taux d'émission dans le vide $\Gamma_{\mathrm{O}}$, est égal au rapport $\mathrm{P} / \mathrm{P}_{\mathrm{O}}$ des puissances rayonnées par un dipôle classique dans les mêmes conditions. En pratique, l'influence d'un résonateur optique sur le temps de vie d'un émetteur, $\Gamma / \Gamma_{\mathrm{o}}$, est généralement décrite par le facteur de Purcell :

$\mathrm{F}_{\mathrm{p}}=\left(3 / 4 \pi^{2}\right) \times(\lambda / \mathrm{n})^{3} \times(\mathrm{Q} / \mathrm{V})$, où $\lambda$ est la longueur d'onde de l'émission, $n$ l'indice de réfraction du milieu environnant, $Q$ est le facteur de qualité du résonateur (fréquence de résonance divisée par la finesse de sa bande passante en fréquence), et $\mathrm{V}$ est le volume du mode de la cavité [4], qui peut être calculé en électrodynamique classique par le facteur $\mathrm{P} / \mathrm{P}_{\mathrm{o}}$.

\section{Des résonateurs optiques à larges bandes et nanométriques}

L'optimisation des résonateurs optiques a donc longtemps porté sur la minimisation de leur finesse spectrale (pour augmenter Q) et de leur taille (pour réduire V), avec le développement de cavités FabryPérot de quelques microns de diamètre mais aussi de résonateurs à modes de galerie ou de cavités à cristal photonique, qui peuvent être réalisés en exploitant les avancées technologiques de la micro et de la nano-électronique [5]. Ces différentes microcavités présentent des volumes effectifs proches de $\lambda^{3}$ et des finesses n'excédant pas quelques centaines de $\mathrm{MHz}$.

Mais l'élargissement spectral de l'émission spontanée, induit par le milieu environnant l'émetteur à température ambiante, atteint plusieurs $\mathrm{THz}$; ceci entraine un recouvrement en fréquence négligeable avec des microcavités optimisées, qui sont mieux adaptées pour se coupler à des sources de lumière en conditions cryogéniques (température de l'ordre du degré kelvin). 
Les antennes optiques proposent alors une stratégie alternative pour concevoir des résonateurs optiques à large bande. En pratique, la largeur spectrale de la résonance doit être augmentée et donc son facteur de qualité réduit. Mais, pour ne pas diminuer $\mathrm{F}_{\mathrm{p}}$, le volume des modes de la cavité qui se couplent à l'émetteur doit être drastiquement diminué. C'est ici que les particules de taille nanométrique, dont le nuage électronique se polarise de manière résonante en présence d'une onde incidente ou d'un émetteur local, démontrent tout leur intérêt. Ces résonances ont des largeurs spectrales similaires aux sources de lumière à température ambiante, et le volume du résonateur est inférieur de plusieurs ordres de grandeur au cube de la longueur d'onde. Toutefois, dans le cas des antennes optiques, la définition du volume des modes de la cavité couplés à l'émetteur a nécessité un travail théorique récent, car ces antennes présentent des pertes par diffusion et par absorption importantes [4].

\section{Des antennes en or et en silicium}

Les matériaux généralement utilisés pour les microcavités optiques, comme la silice ou le nitrure de silicium, ne permettent pas de produire des résonateurs nanométriques. En effet, pour que la polarisabilité d'un matériau bien plus petit que la longueur d'onde présente une résonance, il doit être caractérisé par une permittivité diélectrique réelle négative. Dans le visible, les matériaux les mieux adaptés sont les métaux nobles qui présentent des résonances plasmons, c'està-dire des oscillations collectives de leurs électrons de conduction. En particulier, l'or a plusieurs atouts qui l'ont placé au centre de nombreux développements d'antennes optiques (fig. 1) : les particules d'or, de $5 \mathrm{~nm}$ à $200 \mathrm{~nm}$ de dimension caractéristique, présentent des résonances plasmons dans le visible et le proche infrarouge ; la réactivité chimique de l'or est faible (pas d'oxydation, faible toxicité), mais les nanoparticules d'or peuvent être aisément fonctionnalisées avec des molécules présentant des fonctions thiols.

Quand la taille d'une particule devient de l'ordre de la longueur d'onde, elle peut également présenter une polarisabilité induite résonante avec une permittivité diélectrique positive tant que celle-ci présente un contraste important vis-à-vis de l'environnement, ce qui est le cas des semi-conducteurs dans l'air ou l'eau aux fréquences optiques. Les particules de silicium, avec un diamètre variant entre $100 \mathrm{~nm}$ et $500 \mathrm{~nm}$, sont particulièrement intéressantes car elles présentent des résonances d'ordres faibles (dipolaires et quadripolaires) dans le spectre visible et proche infrarouge, et des pertes ohmiques négligeables par rapport aux métaux nobles. De plus, les modes induits peuvent être électriques ou magnétiques, ce qui n'est pas le cas pour des particules sphériques métalliques dont les résonances plasmons ne sont qu'électriques. Ces résonateurs diélectriques peuvent donc influer sur certains ions luminescents, comme l'europium et l'erbium, qui rayonnent comme des dipôles magnétiques [6]. Surtout, l'utilisation de silicium rend la fabrication de ces résonateurs compatibles avec les techniques standard de la microélectronique.

\section{Concevoir des antennes optiques pour mieux émettre ou absorber la lumière}

Le théorème de réciprocité en électromagnétisme implique qu'une antenne capable d'exalter la réception d'une onde par un matériau polarisable permettra également à ce matériau de rayonner, à la même fréquence, plus intensément en champ lointain. C'est pourquoi les antennes optiques peuvent devenir des objets versatiles pour améliorer l'absorption et l'émission de photons et ainsi optimiser des sources de lumière, mais aussi la conversion d'énergie optique en courant électrique pour des détecteurs ou des cellules solaires [2]. Toutefois, les matériaux utilisés pour concevoir des antennes optiques absorbent partiellement la lumière. Ceci signifie qu'à l'inverse des métaux en radiofréquences, considérés comme parfaitement conducteurs, le champ électromagnétique va pénétrer dans les résonateurs et être partiellement dissipé sous forme de chaleur. Les antennes optiques génèrent donc des pertes ohmiques et l'une des problématiques actuelles est de les réduire.

La brillance d'une source décrite par un système à trois niveaux d'énergie est alors liée à sa capacité d'absorption (section efficace d'excitation), son temps de vie dans l'état excité (inverse de $\Gamma$ ) et le rendement avec lequel un quantum d'énergie absorbé est réémis (rendement quantique d'émission). Une antenne optique va influencer ces trois paramètres : elle va exalter l'intensité locale et augmenter la probabilité d'excitation ; l'émission de photons depuis l'état excité va être accélérée ; et le rendement quantique final va être dominé par l'efficacité de rayonnement de l'antenne vis-à-vis de ses pertes ohmiques. Si la dissipation thermique domine la réponse de l'antenne, l'absorption de photons peut être augmentée avec une émission de photons accélérée, mais les pertes ohmiques entraîneront au final une diminution du signal lumineux (on parle alors de “quenching” de fluorescence). Au contraire, une antenne optimisée pour réduire la dissipation thermique va également augmenter l'efficacité d'excitation et réduire le temps de vie, mais avec une bonne efficacité d'émission pouvant conduire à une nette augmentation du rendement quantique.

À l'image des antennes radiofréquences, les antennes optiques permettent également de contrôler le diagramme de rayonnement d'une source de champ électromagnétique. Ceci permet d'optimiser la directivité des photons émis et donc leur future collection par un système optique ou, par réciprocité, la réception du champ incident et l'excitation de la source. En pratique, la directivité est améliorée en modulant les phases des polarisabilités induites dans les éléments de l'antenne afin de contrôler l'interférence en champ lointain de leur rayonnement et de la rendre constructive dans les angles solides désirés.

Une ingénierie des antennes optiques, et de leur couplage avec la matière condensée, est donc nécessaire afin de contrôler les processus de rayonnement tout en minimisant les pertes ohmiques qui peuvent détruire l'émission de lumière ou limiter la conversion photon / électron dans les dispositifs optoélectroniques. Cette ingénierie nécessite de contrôler la composition et la géométrie des antennes optiques à des échelles bien inférieures à la longueur d'onde de la lumière, soit seulement quelques nanomètres.

\〉 
\〉>

\section{Graver ou organiser la matière à l'échelle du nanomètre pour optimiser l'émission spontanée}

Les problématiques de fabrication d'éléments nanométriques de métaux nobles ou de semi-conducteurs expliquent pourquoi l'essor des antennes optiques au cours de la dernière décennie a été concomitant à des progrès spectaculaires dans la structuration des matériaux inorganiques. On différencie typiquement deux approches : les techniques lithographiques, où un motif nanométrique est gravé dans une résine par un faisceau de photons ou d'électrons avant d'être transféré sur un matériau inorganique, ou usiné directement dans le matériau massif par des ions focalisés; et les méthodes d'auto-assemblage, où des nanoparticules sont associées entre elles par des liaisons ioniques ou moléculaires. Si la lithographie apporte une grande flexibilité dans la forme et la position relative de nanostructures gravées (fig. 2-a) avec une résolution pouvant descendre à $10 \mathrm{~nm}$, l'auto-assemblage permet d'atteindre des distances interparticules contrôlées au nanomètre près, tout en combinant des nanoparticules et des molécules de natures différentes (fig. 2-b). En particulier, l'ADN est de plus en plus utilisé comme un échafaudage programmable permettant d'assembler des milliards de copies d'une même nanostructure en parallèle, et surtout d'y adjoindre un nombre contrôlé de molécules fluorescentes grâce aux avancées récentes des biotechnologies.
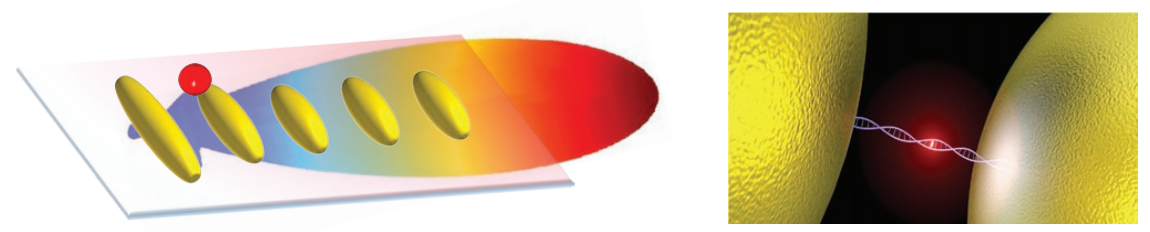

a
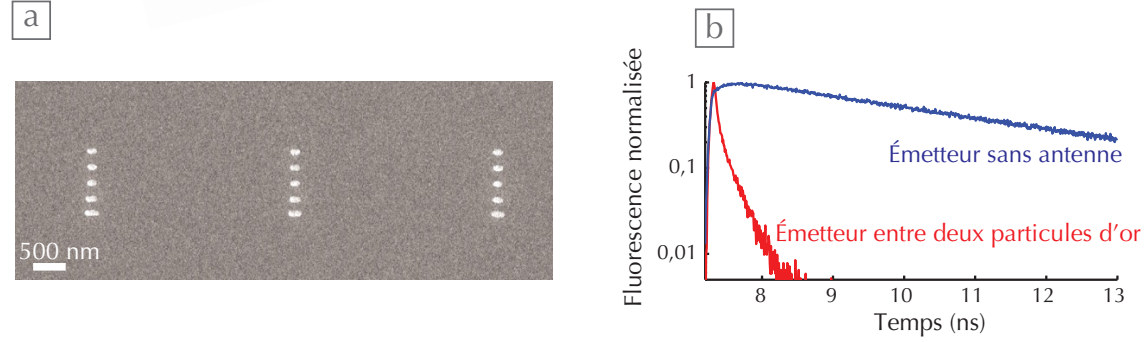

2. Exemples d'antennes optiques lithographiées et auto-assemblées.

(a) Une antenne optique, inspirée par les antennes radiofréquences de type Yagi Uda et couplée à une particule luminescente de semi-conducteur, permet de diriger la lumière émise dans un demi-espace (l'image de microscopie électronique, en bas, montre trois exemplaires de cette antenne fabriqués sur un substrat). (b) Dimère de particules d'or liées par un brin d'ADN sur lequel une molécule fluorescente est greffée : son taux d'émission $\Gamma$ augmente d'un facteur 100 en présence de l'antenne (mesure sur émetteur unique en bas à droite).

\section{Des applications potentielles en optoélectronique}

L'exaltation locale du champ électromagnétique et l'optimisation de la réception du champ incident permettent également d'utiliser les antennes optiques pour réduire la taille des éléments absorbants de photodétecteurs ou de cellules solaires [2]. En effet, obtenir un rendement de conversion photon / électron proche de 1 pour le silicium nécessite des voxels (pixels volumétriques) de plusieurs dizaines de microns de côté. Dans le cas des détecteurs, cette taille introduit une gigue ("jitter"), c'est-à-dire des fluctuations du signal de plusieurs dizaines de picosecondes, qui limite drastiquement la vitesse de réponse des photodiodes. Réduire la taille du détecteur tout en conservant une efficacité d'excitation élevée est donc crucial pour descendre la résolution temporelle de la détection optique aux échelles femtosecondes.

En ce qui concerne les cellules photovoltaïques, la miniaturisation du matériau actif permettant la conversion photon / électron peut avoir un intérêt en terme de coût pour utiliser des semi-conducteurs plus efficaces que le silicium mais, surtout, pour l'utilisation de matériaux moins purs et plus économiques comme le silicium amorphe, les matériaux organiques ou les pérovskites (oxydes de formule $\mathrm{ABO}_{3}$ ) qui présentent des libres parcours de charges réduits [2].

Toutefois les pertes ohmiques des antennes en métaux nobles comme l'or ou l'argent sont très préjudiciables au rendement final de la cellule, non seulement en dissipant l'énergie incidente mais aussi en augmentant la température locale qui réduit le rendement intrinsèque du matériau actif. De plus, ces matériaux ne sont pas compatibles avec les technologies CMOS (Complementary Metal Oxide Semiconductor) utilisées dans la conception des transistors en microélectronique.

C'est pourquoi le développement d'antennes optiques fabriquées avec des semi-conducteurs à haut indice, présentant de plus faibles dissipations thermiques, a un grand potentiel pour remplacer les métaux nobles dans les futures applications optoélectroniques. 


\section{Les äntennes optiques en biotechnologie}

La capacité des antennes optiques à exalter une intensité de fluorescence de plus de trois ordres de grandeur trouve des applications importantes pour la détection de molécules individuelles dans des conditions physiologiques. Ainsi, pour suivre une molécule fluorescente individuelle au sein d'un ensemble de molécules en solution, la concentration en éléments fluorescents ne doit pas dépasser quelques nanomoles par litre. Cette borne supérieure est nécessaire pour s'assurer que le nombre d'émetteurs dans un volume de mesure optique, de l'ordre de la longueur d'onde au cube, est compris entre 0 et 1 . Toutefois, les molécules intervenant dans des réactions biochimiques nécessitent généralement des concentrations plus proches de la micromole par litre. S'il est aujourd'hui possible de marquer des biomolécules comme des brins d'ADN ou des protéines avec des éléments fluorescents, leur forte concentration locale empêche leur analyse, à l'échelle de la molécule individuelle, en conditions physiologiques. Des nanoantennes optiques ont ainsi été développées afin de diminuer le volume de mesure et d'exalter localement le signal d'une molécule tout en minimisant la fluorescence environnante, ce qui a permis de détecter un brin d'ADN individuel dans une solution où sa concentration dépasse plusieurs dizaines de micromoles par litre [7].

Le développement récent des techniques d'auto-assemblage pour la conception d'antennes optiques permet de travailler sur des applications biotechnologiques en diagnostic et en biophysique. En effet, la géométrie d'une antenne influence directement sa réponse optique : par exemple, modifier la position relative de deux particules d'or à l'échelle du nanomètre influence directement la fréquence de résonance de l'antenne et donc la couleur de la lumière qu'elle diffuse. Comme le montre la figure 3, la déformation d'un dimère de particules d'or, liées par un court brin d'ADN, peut être directement suivie, sous microscope, avec une lampe halogène et une caméra couleur [10]. Ceci permet donc de mesurer des distances bien inférieures à la longueur d'onde qui seraient inaccessibles par microscopie classique à cause de la
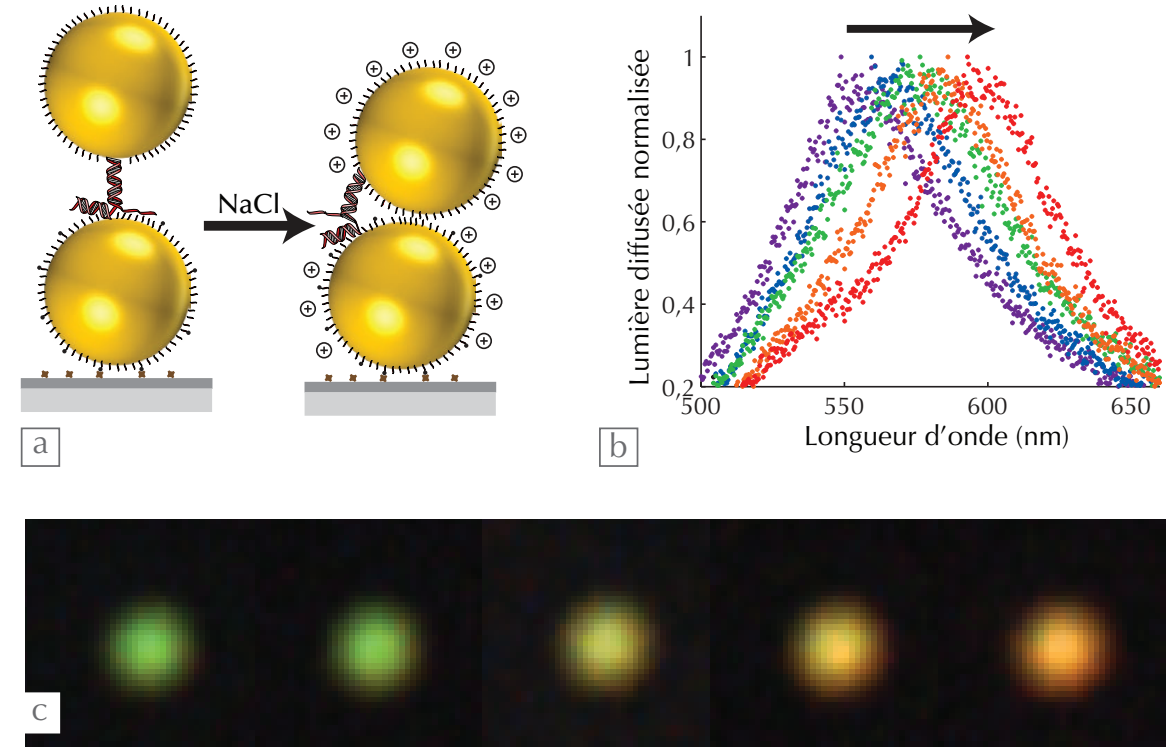

3. Suivi de la déformation nanométrique d'une antenne optique. La distance entre deux particules d'or de $40 \mathrm{~nm}$ varie de $20 \mathrm{~nm}$ à $3 \mathrm{~nm}$ en modifiant la force ionique locale (a), entrainnant un décalage spectral vers le rouge de la résonance plasmon mesurée sur un dimère unique de particules d'or (b), et un changement de couleur visible sur une simple caméra couleur (c).

limite de résolution, avec une résolution temporelle de l'ordre de la microseconde. Il est ainsi possible d'analyser optiquement la géométrie d'un court brin d'ADN unique : que ce soit pour l'étude biophysique de ses fluctuations mécaniques ou pour détecter un changement de conformation induit par une protéine spécifique et d'en détecter ainsi la présence à l'échelle de la molécule unique.

\section{Conclusion}

La capacité des antennes optiques à exalter l'interaction lumière-matière à des échelles nanométriques ouvre donc de nombreuses applications pour optimiser les sources de lumière, les photodétecteurs ou les cellules solaires, mais également pour développer des approches originales en biotechnologie. L'industrialisation de ces applications nécessite toutefois un perfectionnement de la nanofabrication par gravure lithographique ou par auto-assemblage de nanoparticules inorganiques, tant en termes de reproductibilité que de coût. Les travaux récents en nanotechnologie à base de brins d'ADN sont d'ailleurs particulièrement intéressants pour les antennes optiques, grâce à une programmabilité de la morphologie de cet échafaudage à l'échelle du nanomètre et de sa sensibilité à l'environnement chimique local.
Une autre direction de recherche récente, avec un potentiel important en optoélectronique, est le développement d'antennes optiques conçues dans des matériaux semi-conducteurs à indice élevé de réfraction optique.

L'ensemble de ces travaux va permettre aux antennes optiques d'évoluer d'un objet de recherche fondamentale vers un outil technologique flexible pour l'ingénierie de la réception ou de l'émission de photons par la matière condensée à température ambiante.

\section{Références}

1• J. J. Greffet et al., Phys. Rev. Lett. 105 (2010) 117701.

2• L. Novotny, Physics Today 64 (2011) 47-52.

3• L. Novotny et N. van Hulst, Nature Photon. $\mathbf{5}$ (2011) 83.

4• C. Sauvan et al., Phys. Rev. Lett. 110 (2013) 237401.

5• I. Abram et al., Reflets de la Physique 21 / Le Bup 927 (2010) 26.

6• B. Rolly et al., Phys. Rev. B 85 (2012) 245432.

7• D. Punj et al., Nat. Nanotechnol. 8 (2013) 512.

8• M. P. Busson et al., Nat. Commun. 3 (2012) 962.

9• A.G. Curto et al., Science 329 (2010) 930.

10• L. Lermusiaux et al., ACS Nano 9 (2015) 978. 